Artículo

\title{
Diagnóstico de poblaciones silvestres de calabacilla loca en el Altiplano Central de México
}

\author{
Gómez González Adrián ${ }^{1}$ \\ Rangel Guerrero Juan Manuel ${ }^{1}$ \\ Morales Flores Francisco ${ }^{1}$ \\ Aquino Pérez Gildardo ${ }^{1}$ \\ Santana García Mario Arturo ${ }^{2}$ \\ Silos Espino Héctor ${ }^{2 \S}$
}

${ }^{1}$ Colegio de Postgraduados-Campus San Luis Potosí. Iturbide 73, Salinas de Hidalgo, San Luis Potosí. CP. 76800. (agomez@colpos.mx; agomez@colpos.mx; franciscojmf@colpos.mx; jaquino@colpos.mx) ${ }^{2}$ Laboratorio de Cultivo de Tejidos Vegetales-Instituto Tecnológico El Llano. Carretera AguascalientesSan Luis Potosí km 18, El Llano, Aguascalientes, México. CP. 20330. (santanam@ produce.net.mx).

${ }^{\S}$ Autor para correspondencia: silosespino@ hotmail.com.

\section{Resumen}

La especie, Cucurbita foetidissima Kunth (calabacilla loca) crece en sitios poco propicios para la agricultura y tiene importancia agroindustrial, por lo anterior, se precisa el conocer su situación actual para proponer en un futuro si la planta se aprovecha de forma sustentable. Por lo anterior se analizaron variables morfológicas (follaje y raíz) de poblaciones ubicadas en la región comprendida entre los Estados de San Luis Potosí, Jalisco y Zacatecas. Las plantas de La Cócona y Yoliatl San Luis Potosí, contienen más guías por planta (9-12), mejores atributos de la raíz, en peso (2536 g), número de guías (67) y volumen $\left(2411.2 \mathrm{~cm}^{3}\right)$ y más alto contenido de frutos. Las plantas de Guadalupe y Ojocaliente Zacatecas mostraron mayor longitud de guías (16-27), longitud del peciolo $(7.5 \mathrm{a} 12 \mathrm{~cm})$ y hojas más grandes $(13.5 \mathrm{a} 18 \mathrm{~cm})$. Respecto a las plantas de Chinampas Ojuelos, Encarnación de Díaz y Lagos de Moreno Jalisco, estas presentaron en raíz un $1615 \mathrm{~g}$ de peso, $33.8 \mathrm{~cm}$ de perímetro, $10.8 \mathrm{~cm}$ de amplitud, $52.6 \mathrm{~cm}$ de longitud, 44.5 guías, $1690.5 \mathrm{~cm}^{3}$ de volumen y 162 frutos. Estos bajos atributos pueden deberse a que las plantas se ubicaron en sitios caracterizados por climas más cálidos y con mayor precipitación (473.5- 573.2 $\mathrm{mm}$ ) en comparación a las otras regiones. En este trabajo se estableció la geo-referenciación y el tamaño de las plantas silvestres de calabacilla loca como una primera estrategia para conocer su condición del hábitat, definir una alternativa de conservación/propagación y proponer un método de aprovechamiento sustentable de acuerdo con su potencial agroindustrial que se le conoce.

Palabras claves: cucurbita foetidissima Kunth, caracterización, descripción botánica, estadística multivariada.

Recibido: mayo de 2019

Aceptado: julio de 2019 


\section{Introducción}

El uso integral y sustentable de plantas ruderales, arvenses, ornamentales y cultivadas puede incrementar los ingresos de los agricultores de áreas marginales del altiplano central de México (Juárez-Pérez et al., 1991). Una de estas plantas puede ser la especie, Cucurbita foetidissima Kunth, calabacilla loca que crece en sitios poco propicios para la agricultura (tolerante a altas temperaturas, resistente a plagas y enfermedades), la raíz contiene carbohidratos de potencial industrial $23 \%$ amilosa), las semillas contienen hasta $33.9 \%$ de aceite, buen contenido de proteína $34.2 \%$, las hojas pueden consumirse para forraje y ofrece muchas posibilidades como fuente de alimento (Granados-Sánchez y López-Ríos, 1999; Hoover, 2001).

Específicamente, por sus principales componentes, según Ordoñes (2007) sugirieron que las hojas pueden utilizarse como forraje por su alta digestibilidad (75-85\%) en cabras y como alimento humano por su contenido de aceite en semilla, Calvo-Grajales (2003) determinó que contiene ácidos grasos palmítico, esteárico, linoleico y oleico y tienen acción similar a los aceites comerciales para cocinar (aún sin utilizar antioxidantes).

La especie se encuentra distribuida en Guanajuato, Hidalgo, Querétaro, Jalisco, San Luis Potosí y Zacatecas, así como el sur de los Estados Unidos de América (Villaseñor y Espinoza, 1998; Hernández et al., 2000; Lira et al., 2009). Por lo anterior, se precisa investigar, caracterizar y generar modelos sobre los caracteres vegetales sobresalientes que permitan conocer el crecimiento y desarrollo de la especie para hacer más eficientes los usos conocidos. Finalmente proponer un aprovechamiento sustentable y definir estrategias para su conservación.

\section{Materiales y métodos}

\section{Área de estudio}

El área de estudio abarca la región comprendida entre los estados de San Luis Potosí (Salinas, Villa de Reyes y Villa de Ramos), Jalisco (Ojuelos, Encarnación de Díaz, Lagos de Moreno) y Zacatecas (Guadalupe, Ojocaliente y La Pendencia Pinos). En cada sitio se realizaron recorridos de campo, alrededor de las cabeceras municipales y preferentemente en sitios de fácil acceso.

\section{Características de la zona de estudio}

En esta zona convergen cuatro regiones fisiográficas: la Altiplanicie Septentrional, en el centro y norte de México; la Altiplanicie Meridional, en el sur y oriente, las Sierras Inferiores, al noreste y las Sierras Altas con Valles, al occidente del área (Tamayo, 1981). Existen serranías y llanuras, con altitudes de 1000 a 2600 msnm. (Ferrusquía-Villafranca, 1993) y amplias zonas de aluvión con cerros aislados.

El sustrato más común en el norte del área son las rocas calizas del Cretácico y del Jurásico; en el sur y oeste son más comunes las rocas ígneas, en su mayoría riolitas y en ocasiones andesitas y basaltos. Predominan suelos tipo Calcisol, distinguidos por la acumulación de carbonato de calcio;

en el noreste existen zonas de Regosol, suelos poco profundos y de baja fertilidad (Cuanalo et al., 1989; Ferrusquía-Villafranca, 1993). 
El clima que predomina es el BS, con las variantes BS0, el más seco y BS1, el menos seco (García, 1973). La precipitación es escasa e irregular, con un promedio anual de 240 a $770 \mathrm{~mm}$ (Cuanalo et al., 1989). La temperatura media anual varía de 13.5 a $20.5^{\circ} \mathrm{C}$ (Anónimo, 1980).

\section{Determinación de tamaño de muestra}

Previo a la recolecta general, se realizó un muestreo piloto durante los días 19 y 27 de marzo de 2014, en dos sitios: la Cocona, Salinas de Hidalgo San Luis Potosí y Chinampas, Ojuelos Jalisco. Con los datos obtenidos se calculó el tamaño de muestra real. El número de plantas a muestrear se obtuvo usando la fórmula de Bolflor et al. (2000). Donde: $\mathrm{n}=$ número de plantas muestreadas; $\mathrm{E}=$ margen de error que se permite para esta colecta; $t=$ valor de ' $t$ ' de student $(t=0.05)$; $N=$ total de población de plantas de calabacilla loca en la localidad; $\mathrm{CV}=$ coeficiente de variación; para obtener este valor es necesario hacer un muestreo piloto.

$\mathrm{n}=\frac{\mathrm{t}^{2} * \mathrm{CV}^{2}}{\mathrm{E}^{2}+\frac{\mathrm{t}^{2} * C V^{2}}{\mathrm{~N}}}$

\section{Descriptores analizados}

En los sitios seleccionados se muestrearon 25 plantas por cada uno de ellos y se tomaron datos de los siguientes descriptores morfológicos: en relación al follaje, 1) se analizaron número (NPG) y longitud de las guías por planta (LGP), este último dato se midió en $\mathrm{cm}$ desde el inicio del tallo hasta el ápice de la última hoja, distancia entre entrenudos; y 2) hojas, considerando la longitud de hoja (LH), amplitud de la hoja (AH) y longitud del peciolo (LP), medidas en unidades de $\mathrm{cm}$. Con relación a la raíz se determinó la longitud, amplitud y diámetro $(\mathrm{cm})$ y volumen expresado en $\mathrm{cm}^{3}$. Para estos últimos se extrajeron tres plantas de cada sitio, las cuales contenían 3, 8 y 13 guías.

\section{Indicadores para determinar el estado de desarrollo de las plantas}

De acuerdo a la Figura 1 se determinaron los agrupamientos de las plantas de calabacilla loca.

\section{Análisis de datos morfológicos}

Los datos de variables morfológicas simples se integraron en intervalos de acuerdo al comportamiento de crecimiento y se analizaron por medio de comparación de promedios entre los sitios de poblaciones silvestres.

\section{Agrupamiento de Cucurbita foetidissima Kunt acorde a componentes morfológicos}

Para realizar el agrupamiento por componentes principales se analizaron 13 variables morfológicas de plantas de Cucurbita foetidissima Kunth, integradas en caracteres vegetativos (distancias entrenudos, el largo y amplitud de la hoja, así como el largo del peciolo, número de guías totales, la longitud de las guías), de desarrollo reproductivo (número de flores y número de frutos) y de la raíz (peso total, volumen, longitud, amplitud ancho, el largo). 


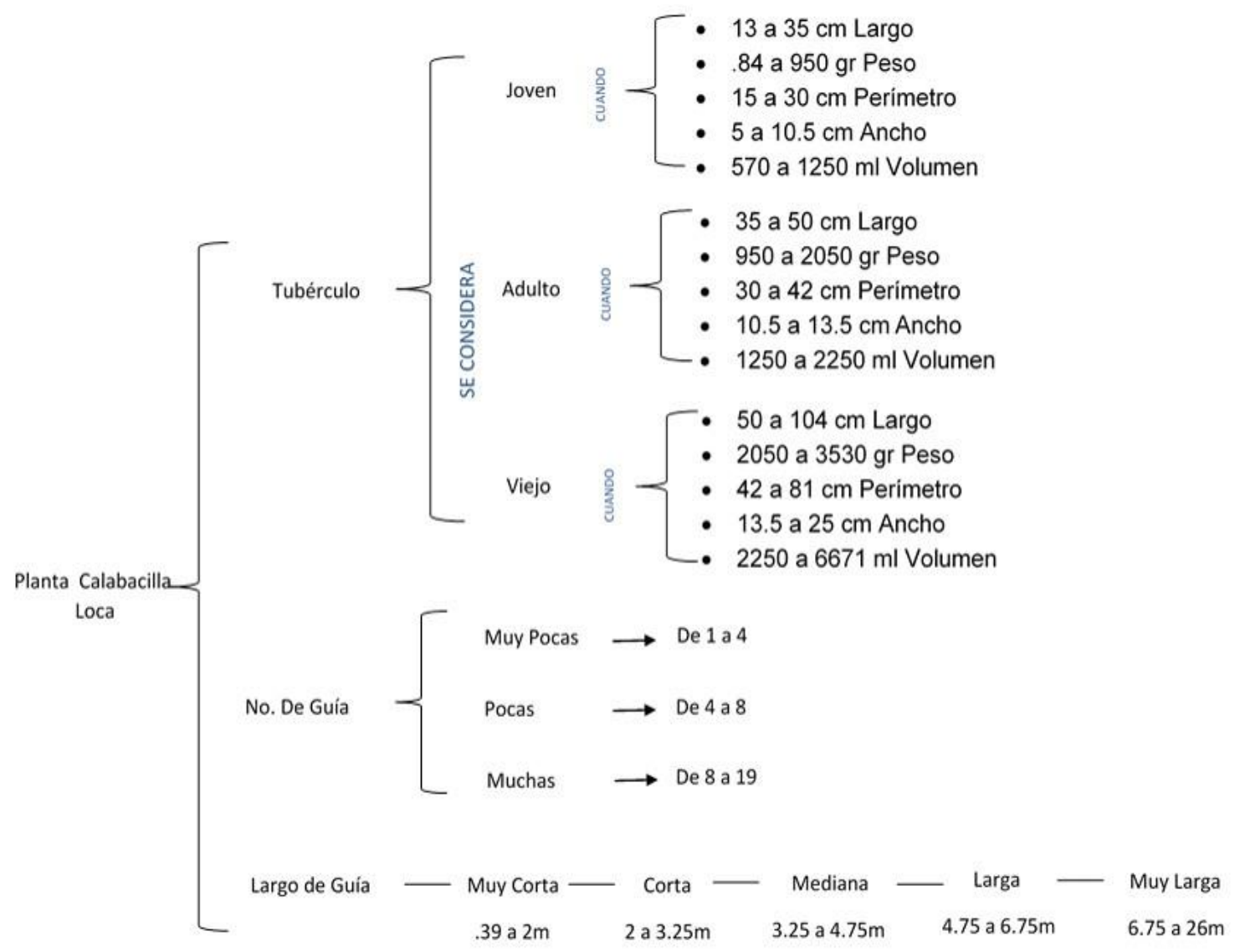

Figura 1. Indicadores que permiten identificar el estado de desarrollo de la planta. Humán (1991). Descriptores de la batata. CIP/AVRDC/IBPGR.

Con la base de datos se realizó un análisis multivariado basado en análisis de componentes principales (ACP) para disminución de inferencia y agrupamiento por similaridad para comprender la información de grupos de plantas con características similares, discriminando grupos usando la varianza interna mínima (Ward) para formar tipos de plantas, se utilizó Infostat (Di Rienzo, 2014).

\section{Resultados y discusión}

\section{Geo-referenciación de los hábitats de calabacilla loca}

Por lo general las zonas muestreadas se encontraron en orillas de carreteras o campos de cultivo, con una pendiente entre 15 y $45^{\circ} \mathrm{C}$ y una altitud de 1814 y $2475 \mathrm{msnm}$. Se mencionan las coordenadas, altitud y características de cada localidad de colecta. Las comunidades vegetales que encontramos fueron condiciones diferentes de acuerdo a la región y a las características del lugar de colecta (Figura 2 y Cuadro 1). 

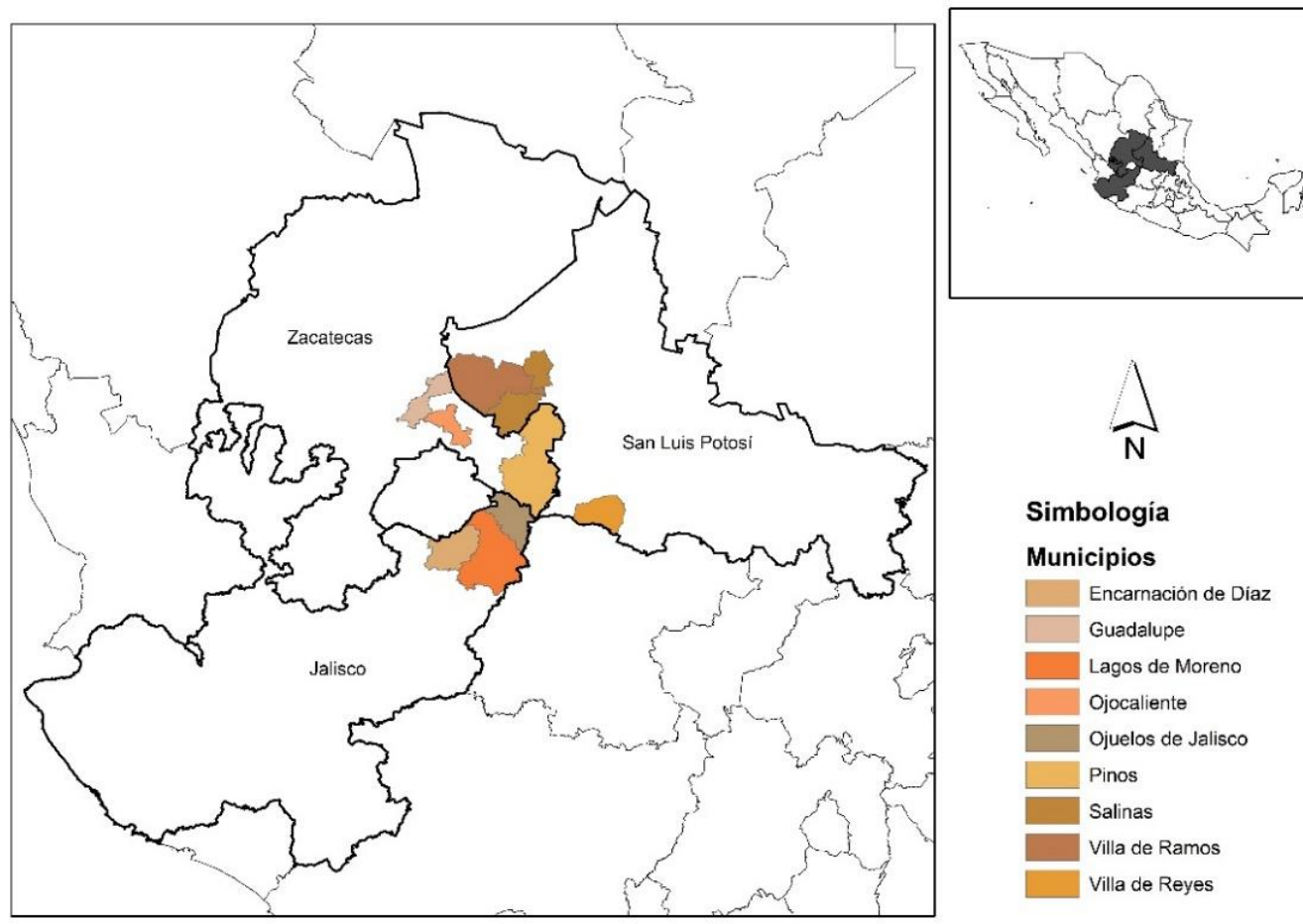

Figura 2. Municipios donde se realizó el estudio de calabacilla loca.

Cuadro1. Ubicación donde se encontraron poblaciones silvestres de Cucurbita foetidissima Kunth.

\begin{tabular}{|c|c|c|c|c|c|}
\hline Estado & Localidad & Coordenadas & $\begin{array}{l}\text { Altitud } \\
\text { (m) }\end{array}$ & $\begin{array}{l}\text { Precipitación } \\
\text { pluvial }(\mathrm{mm})\end{array}$ & $\begin{array}{l}\text { Características del lugar } \\
\text { de colecta }\end{array}$ \\
\hline \multirow[t]{3}{*}{$\begin{array}{l}\text { San Luis } \\
\text { Potosí }\end{array}$} & $\begin{array}{l}\text { La Cocona, } \\
\text { Salinas }\end{array}$ & $\begin{array}{c}21^{\circ} 48^{\prime} 5.1^{\prime \prime} \mathrm{N} \\
101^{\circ} 48^{\prime} 55.6 " \mathrm{O}\end{array}$ & 2099 & 391 & $\begin{array}{l}\text { Orilla de carretera, } \\
\text { cabecera de parcela }\end{array}$ \\
\hline & $\begin{array}{l}\text { El Centenario, } \\
\text { Villa de Reyes }\end{array}$ & $\begin{array}{l}21^{\circ} 46^{\prime} 38.6 ” \mathrm{~N} \\
100^{\circ} 56^{\prime} 58.3 ” \mathrm{O}\end{array}$ & 1814 & 360 & $\begin{array}{l}\text { Borde de carretera, } \\
\text { borde de arroyo }\end{array}$ \\
\hline & $\begin{array}{l}\text { Yoliatl, Villa de } \\
\text { Ramos }\end{array}$ & $\begin{array}{l}22^{\circ} 59^{\prime} 4.56^{\prime \prime} \mathrm{N} \\
101^{\circ} 54^{\prime} 38.0^{\prime \prime} \mathrm{O}\end{array}$ & 2190 & 429.6 & $\begin{array}{l}\text { Orilla de carretera, } \\
\text { costado de tierras de } \\
\text { cultivo }\end{array}$ \\
\hline \multirow[t]{3}{*}{ Jalisco } & $\begin{array}{l}\text { Chinampas, } \\
\text { Ojuelos }\end{array}$ & $\begin{array}{l}21^{\circ} 48^{\prime} 5.12^{\prime \prime} \mathrm{N} \\
101^{\circ} 48,55.6 " \mathrm{O}\end{array}$ & 2095 & 473.5 & $\begin{array}{l}\text { Orilla de carretera, } \\
\text { arroyo de aguas negras } \\
\text { y a un costado de tierras } \\
\text { de cultivo }\end{array}$ \\
\hline & $\begin{array}{l}\text { Encarnación de } \\
\text { Díaz }\end{array}$ & $\begin{array}{l}21^{\circ} 38^{\prime} 48.44^{\prime \prime} \mathrm{N} \\
102^{\circ} 16^{\prime} 40.4^{\prime \prime} \mathrm{O}\end{array}$ & 1851 & 564 & $\begin{array}{l}\text { Orilla de carretera en } \\
\text { terreno con grava }\end{array}$ \\
\hline & Lagos de Moreno & $\begin{array}{l}19^{\circ} 50^{\prime} \mathrm{N} \\
10359^{\prime} \mathrm{O}\end{array}$ & 1876 & 573.2 & $\begin{array}{l}\text { En medio de la } \\
\text { autopista, a lado de } \\
\text { terreno de agostadero }\end{array}$ \\
\hline
\end{tabular}




\begin{tabular}{ccccc}
\hline Estado & Localidad & Coordenadas & $\begin{array}{c}\text { Altitud } \\
(\mathrm{m})\end{array}$ & $\begin{array}{l}\text { Precipitación Características del lugar } \\
\text { pluvial }(\mathrm{mm})^{*}\end{array}$ \\
\hline Zacatecas colecta
\end{tabular}

De acuerdo con Anónimo (2016); ${ }^{*}=$ documentos por municipio de los gobiernos de los estados de San Luis Potosí, Jalisco y Zacatecas.

Comportamiento de variables morfológicas (guías y hojas). De manera general, en el Cuadro 2, se indican los valores cuantitativos del crecimiento vegetativo de un total de 25 plantas analizadas en nueve sitios de plantas de Cucurbita foetidissima Kunth. Se observó que existe una gran variación en el número de guías por planta (1-19), predominan las plantas con menos de ocho guías, las plantas de Chinampas Ojuelos y de manera sobresaliente, las plantas ubicadas en La Cócona, Salinas de Hidalgo y Yoliatl Villa de Ramos. San Luis Potosí, mostraron más guías (9-12) probablemente a mayor número de años o condiciones climáticas menos favorables (requerimiento natural de la planta).

Cuadro 2. Descripción del crecimiento vegetativo de las plantas de Cucurbita foetidissima Kunth del Altiplano Central de México.

\begin{tabular}{|c|c|c|c|c|c|c|c|c|c|}
\hline \multirow{2}{*}{ Variable } & \multicolumn{2}{|c|}{ Jalisco } & \multicolumn{3}{|c|}{ San Luis Potosí } & \multicolumn{3}{|c|}{ Zacatecas } & \multirow{2}{*}{ Subtotal/grupo } \\
\hline & $\mathrm{a}$ & $\mathrm{b}$ & c & $\mathrm{d}$ & $\mathrm{e}$ & $f$ & $\mathrm{~g}$ & $\mathrm{~h}$ & \\
\hline \multicolumn{10}{|l|}{ Guías por planta } \\
\hline De $1-4$ & 14 & 6 & 20 & 4 & 9 & 19 & 6 & 7 & 85 \\
\hline De $5-8$ & 10 & 3 & 2 & 9 & 7 & 6 & 17 & 17 & 71 \\
\hline De 9-19 & 1 & 2 & 3 & 12 & 9 & - & 2 & 1 & 30 \\
\hline Subtotal-región & 25 & 11 & 25 & 25 & 25 & 25 & 25 & 25 & 186 \\
\hline \multicolumn{10}{|l|}{ Longitud de guías } \\
\hline De $0.39-200 \mathrm{~cm}$ & 18 & 2 & 13 & 34 & 1 & 4 & 27 & 6 & 105 \\
\hline De $201-325 \mathrm{~cm}$ & 26 & 4 & 15 & 23 & 8 & 9 & 32 & 14 & 131 \\
\hline De $326-475 \mathrm{~cm}$ & 15 & 13 & 14 & 18 & 15 & 7 & 10 & 27 & 119 \\
\hline De $476-675 \mathrm{~cm}$ & 1 & 9 & 8 & 1 & 34 & 15 & 1 & 20 & 89 \\
\hline De $676 \mathrm{~cm}-26 \mathrm{~m}$ & 1 & - & 5 & - & 12 & 16 & - & 2 & 36 \\
\hline Subtotal-región & 61 & 28 & 55 & 76 & 70 & 51 & 70 & 69 & 480 \\
\hline \multicolumn{10}{|c|}{ Longitud del peciolo $(\mathrm{cm})$} \\
\hline Corto & 12 & 27 & 3 & 1 & 31 & 6 & 1 & 10 & 91 \\
\hline Mediano & 28 & 1 & 21 & 39 & 38 & 25 & 23 & 22 & 197 \\
\hline Largo & 13 & - & 18 & 27 & 1 & 11 & 39 & 15 & 124 \\
\hline Muy largo & 8 & - & 13 & 9 & - & 9 & 7 & 22 & 68 \\
\hline Subtotal-región & 61 & 28 & 55 & 76 & 70 & 51 & 70 & 69 & 480 \\
\hline
\end{tabular}




\begin{tabular}{|c|c|c|c|c|c|c|c|c|c|}
\hline \multirow{2}{*}{ Variable } & \multicolumn{2}{|c|}{ Jalisco } & \multicolumn{3}{|c|}{ San Luis Potosí } & \multicolumn{3}{|c|}{ Zacatecas } & \multirow{2}{*}{ Subtotal/grupo } \\
\hline & $\mathrm{a}$ & $\mathrm{b}$ & $\mathrm{c}$ & $\mathrm{d}$ & $\mathrm{e}$ & $f$ & $\mathrm{~g}$ & $\mathrm{~h}$ & \\
\hline \multicolumn{10}{|c|}{ Longitud de las hojas (cm) } \\
\hline Muy pequeña & 11 & 15 & 7 & 16 & 18 & 7 & 2 & 7 & 83 \\
\hline Pequeña & 20 & 10 & 16 & 22 & 38 & 18 & 9 & 7 & 140 \\
\hline Mediana & 9 & 3 & 15 & 22 & 12 & 12 & 19 & 15 & 107 \\
\hline Grande & 14 & - & 12 & 13 & 2 & 13 & 23 & 25 & 102 \\
\hline Muy grande & 7 & - & 5 & 3 & - & 1 & 17 & 15 & 70 \\
\hline Subtotal-región & 61 & 28 & 55 & 76 & 70 & 51 & 70 & 69 & 480 \\
\hline \multicolumn{10}{|c|}{ Amplitud de las hojas (cm) } \\
\hline Muy pequeña & 14 & 13 & 3 & 11 & 38 & 3 & 1 & 5 & 88 \\
\hline Pequeña & 23 & 14 & 14 & 29 & 26 & 25 & 17 & 20 & 168 \\
\hline Mediana & 6 & - & 6 & 16 & 3 & 10 & 15 & 2 & 58 \\
\hline Grande & 14 & 1 & 26 & 18 & 3 & 13 & 30 & 20 & 125 \\
\hline Muy grande & 4 & - & 6 & 2 & - & - & 7 & 22 & 41 \\
\hline Subtotal/región & 61 & 28 & 55 & 76 & 69 & 51 & 70 & 69 & 480 \\
\hline
\end{tabular}

a= Chinampas; $\mathrm{b}=$ Encarnación de Díaz; $\mathrm{c}=$ Centenario; $\mathrm{d}=$ Cócona; $\mathrm{e}=$ Yolíatl; $\mathrm{f}=$ Guadalupe; $\mathrm{g}=$ La Pendencia; $\mathrm{y}$ h= Ojocaliente. Lagos de Moreno Jalisco, no se reportan datos por efecto de podas a la orilla de carretera.

En longitud de guías preponderaron aquellas con menor a $6.75 \mathrm{~m}$ y las más largas se encontraron en Guadalupe Zacatecas. En longitud del peciolo predominaron las de tamaño grande-mediano $(7.5$ a $12 \mathrm{~cm})$ de largo, aunque las de Ojocaliente sobresalieron por mayor longitud, con suelos poco profundos. En tamaño de la hoja, las plantas mostraron de pequeño a grande $(16.5 \mathrm{a} 27 \mathrm{~cm})$ y de manera sobresaliente, las plantas de Ojocaliente. Presentaron mayor cantidad de hojas más grandes $(13.5$ a $18 \mathrm{~cm})$.

De acuerdo con los sitios de colecta, las plantas de Chinampas, Ojuelos Jalisco, se localizan principalmente a orillas de carreteras y caminos, cohabitan con pastos de rápido crecimiento y a veces son 'podadas de orilla de carretera' por lo que su crecimiento es diferencialmente inducido en relación a plantas de otros sitios analizados.

Las plantas de La Cócona, Salinas de Hidalgo San Luis Potosí. Forman poblaciones aglomeradas con una separación entre plantas muy corta $(0.5-1 \mathrm{~m})$ por lo que existe una alta competencia por los pocos recursos hídricos y minerales que disponen. Las plantas de Encarnación de Díaz Jalisco se encontraron en un montón de grava orilla de carretera, mostraban tener varios años y no competían con alguna maleza, por eso presentan el peciolo más corto.

Las plantas de Guadalupe Zacatecas, compiten con pastos que limita el crecimiento óptimo de la calabacilla, por lo que tienden a desarrollar peciolos más largos para que las hojas obtengan luz solar se encontraron plantas como jaral (Cistus ladanifer), árnica (Arnica angustifolia), escobilla o hierba de la hormiga (Pathenium hysterophorus L.), nopal (Opuntia spp.), cardenche ( $O$. imbricata) y mezquite (Prosopis laevigata) preferentemente, lo que indica una sinergia que conduce a plantas generalmente grandes $(676 \mathrm{~cm})$. 
En Lagos de Moreno Jalisco, las plantas se desarrollan como 'plantas medianas' (326-475 cm). En el sitio El centenario, Villa de Reyes San Luis Potosí, el clima es más húmedo por lo que existen pocas poblaciones de 'Calabacilla Loca' debido a mayor crecimiento de pasto en lugares susceptibles.

En Yoliatl, Villa de Ramos. San Luis Potosí, Cucurbita foetidissima Kunth. Requiere poca agua y crece más rápido en ambientes áridos y semiáridos (Web Academia, 2013). Las plantas que llegan a desarrollarse se ubican en cuna de relieves y cohabitan con plantas como el gigante (Nicotiana glauca), nopal (Opuntia spp.), yuca (Yucca sp.) y pasto muy corto (Boutelova curtipendula). (Anónimo, 2016).

Descripción de los tubérculos de Cucurbita foetidissima Kunth. En general, las plantas de Chinampas (Ojuelos), Encarnación de Díaz y Lagos de Moreno (Jalisco) mostraron en sus variables de raíz, valores intermedios entre las regiones estudiadas (Cuadro 3).

Cuadro 3. Características del tubérculo de Cucurbita foetidissima Kunth.

\begin{tabular}{|c|c|c|c|c|c|c|c|}
\hline \multirow[b]{2}{*}{ Sitio de colecta } & \multicolumn{7}{|c|}{ Variable analizada } \\
\hline & $\begin{array}{c}\text { Peso de } \\
\text { tubérculo (g) }\end{array}$ & $\begin{array}{l}\text { Perímetro } \\
\quad(\mathrm{cm})\end{array}$ & $\begin{array}{l}\text { Amplitud } \\
\text { (cm) }\end{array}$ & $\begin{array}{l}\text { Longitud } \\
\quad(\mathrm{cm})\end{array}$ & Volumen $\left(\mathrm{cm}^{3}\right)$ & $\begin{array}{c}\text { Guías } \\
\text { (numero) }\end{array}$ & $\begin{array}{l}\text { Frutos } \\
\text { por sitio }\end{array}$ \\
\hline \multicolumn{8}{|l|}{ Jalisco } \\
\hline $\begin{array}{l}\text { Chinampas } \\
\text { (Ojuelos) }\end{array}$ & $1403.3(754.1)$ & $30.2(3.4)$ & $10(1.1)$ & $54.8(10.6)$ & $1530(825)$ & 61 & 44 \\
\hline $\begin{array}{l}\text { Encarnación de } \\
\text { Díaz }\end{array}$ & $2368.3(1 \quad 103.1)$ & $44(6.2)$ & $13.6(1.8)$ & $52.6(10.7)$ & $2393.3(1047.4)$ & 28 & 118 \\
\hline Lagos de Moreno & $1073.3(336.5)$ & $27.3(4.7)$ & $8.83(1.6)$ & $50.3(6.6)$ & $1148.3(293)$ & nd & nd \\
\hline Promedio & $1615(902.4)$ & $33.8(8.8)$ & $10.8(2.5)$ & $52.6(8.4)$ & $1690.5(878.1)$ & $23.3^{*}$ & $81^{*}$ \\
\hline \multicolumn{8}{|l|}{ San Luis Potosí } \\
\hline $\begin{array}{l}\text { La Cócona, } \\
\text { Salinas }\end{array}$ & $3560(3881.4)$ & $41.8(19.2)$ & $14.6(7.7)$ & $42.6(16.9)$ & 2977 (3 244.3) & 76 & 116 \\
\hline $\begin{array}{l}\text { Yolíalt, V. de } \\
\text { Ramos }\end{array}$ & $2024(1330.2)$ & $61(20.5)$ & $18.6(6.5)$ & $63(35.5)$ & $2178.3(1$ 169.1) & 70 & 649 \\
\hline $\begin{array}{l}\text { El Centenario, V. } \\
\text { Reyes }\end{array}$ & 2 024(1330.2) & $33.9(12.4)$ & $12.6(4.6)$ & $49.8(21)$ & $2078.3\left(\begin{array}{ll}1 & 015\end{array}\right)$ & 55 & 5 \\
\hline Promedio & $2536(2289.35)$ & $45.5(19.5)$ & $15.3(6.1)$ & $51.8(24)$ & $2411.2(1847.3)$ & $67(10.8)$ & $769(256)$ \\
\hline \multicolumn{8}{|l|}{ Zacatecas } \\
\hline $\begin{array}{l}\text { La Pendencia, } \\
\text { Pinos }\end{array}$ & $836.6(163.5)$ & $17(1)$ & $9.4(0.5)$ & $26(3.6)$ & $859(252.5)$ & 70 & 75 \\
\hline Guadalupe & $1440(677.4)$ & $30(2.9)$ & $11.5(2.6)$ & $31.3(4.5)$ & $1426.6(598.1)$ & 51 & 53 \\
\hline Ojocaliente & $1470(913.2)$ & $30(14.1)$ & $10(4.3)$ & $30(14.7)$ & 2 436.6(1 697.6) & 69 & 90 \\
\hline Promedio & $1248.8(652.4)$ & $25.7(9.7)$ & $10.3(2.7)$ & $29.1(8.2)$ & $1574.1(1$ 142.2) & $63.3(10.6)$ & 218 \\
\hline Promedio general & 1404.8 & 35.03 & 12.15 & 44.52 & 1891.96 & & \\
\hline
\end{tabular}

$\mathrm{Nd}=$ no determinado $;{ }^{*}=$ promedio de dos poblaciones. 
Estas mostraron un peso de $1615 \mathrm{~g}$, perímetro de $33.8 \mathrm{~cm}$, amplitud de $10.8 \mathrm{~cm}$, longitud de 52.6 $\mathrm{cm}$, número de guías de 44.5 , un volumen de $1690.5 \mathrm{~cm}^{3}$ y contenido de frutos de 162 . Lo anterior, puede deberse a que las plantas crecen en condiciones edafo climáticas poco favorables a las ecesidades óptimas de la planta, ya que estos sitios se caracterizan por climas más cálidos y con mayor precipitación (en Chinampas Ojuelos, 473.5 mm, Encarnación de Díaz 564 mm y Lagos de Moreno $573.2 \mathrm{~mm}$ respectivamente).

De manera sobresaliente, las plantas de La Cócona (Salinas), Yoliatl (Villa de Ramos) y el Centenario (Villa de Reyes) correspondientes al Estado de San Luis Potosí, mostraron en promedio, el mayor atributo de la raíz con un peso (2 $536 \mathrm{~g})$, perímetro $(45.5 \mathrm{~cm})$, amplitud (15.3 $\mathrm{cm}$ ), longitud $(51.8 \mathrm{~cm})$, número de guías (67), volumen de $2411.2 \mathrm{~cm}^{3}$ y contenido de frutos de 769 (en su totalidad).

Los factores que posiblemente pueden influir en 'este óptimo desarrollo' pueden ser los siguientes: mayor incidencia de radiación solar, poca competencia con pastos 'agresivos' y menor índice de precipitaciones (La Cocona Salinas con $391 \mathrm{~mm}$, Yoliatl Villa de Ramos con $429.6 \mathrm{~mm}$ y El Centenario Villa de Reyes con $360 \mathrm{~mm}$ respectivamente). Las plantas con menores atributos en las variables de raíz, las encontramos en las en el estado de Zacatecas con un peso de $1248.8 \mathrm{~g}$, perímetro de $25.7 \mathrm{~cm}$, amplitud de $10.3 \mathrm{~cm}$, longitud $29.1 \mathrm{~cm}$, numero de guías de 63.3, un volumen de $1574.1 \mathrm{~cm}^{3}$ y contenido de frutos de 218 .

Los menores atributos de la raíz de esta zona pueden deberse a una mayor altitud sobre el nivel del mar, a menor temperatura media anual, humedad ambiental baja o porque los suelos son menos fértiles o poco profundos, ya que las precipitaciones son intermedias entre las regiones estudiadas (La pendencia, Pinos, $429.6 \mathrm{~mm}$, Guadalupe, $500 \mathrm{~mm}$ y Ojocaliente, $500 \mathrm{~mm}$ respectivamente).

\section{Frutos}

En el número de frutos por sitio (Cuadro 3), Yoliatl sobresalió con 649 frutos en total, seguido por Encarnación de Díaz (118) y La Cocona, Salinas de Hidalgo, San Luis Potosí (116). Aquí dedujimos que el clima es un factor importante en las plantas de calabacilla, ya que encontramos mayor cantidad de frutos en sitios con menor precipitación y mayor incidencia solar.

El número de frutos por sitios es importante y complementa a lo encontrado por Flores (2016) en donde reporta que calabacilla loca de esos lugares presentan 100 semillas un peso de 3.7 g y 27 017 semillas por kilogramo. Por su parte Flores (2016) determinó que los ácidos linoleico y linolénico (ácidos grasos esenciales) que contiene la semilla son de gran valor para su posible uso como aceites comestibles. De acuerdo con lo anterior, el sitio más recomendable para colectar frutos y semilla es Yoliatl en Villa de Ramos, San Luis Potosí.

Estimación del estado de desarrollo de poblaciones de Cucurbita foetidissima Kunth en relación a características morfológicas. Con la información de las variables morfológicas analizadas se realizó una estimación (Cuadro 4) de la condición probable de desarrollo que presentan las poblaciones nativas de calabacilla, infiriendo una clasificación de planta de acuerdo a su porte. Así, generalmente, las plantas de Jalisco que tienen un volumen de raíz de $1690.5 \mathrm{~cm}^{3}$ se consideran de tamaño intermedio, mientras que la de San Luis Potosí con un volumen de raíz de $2411.2 \mathrm{~cm}^{3}$ las plantas son consideradas de porte grande y finalmente, las plantas del Estado de Zacatecas con un volumen de raíz de $1574.1 \mathrm{~cm}^{3}$ las plantas son consideradas pequeñas. 
Cuadro 4. Inferencia de edad de las plantas de calabacilla loca, Cucurbita foetidissima Kunth de acuerdo a variables morfológicas de la raíz.

\begin{tabular}{clccc}
\hline \multirow{2}{*}{ Componente } & Rasgo morfológico & \multicolumn{2}{c}{ Porte de planta de acuerdo con su carácter morfológico } \\
\cline { 3 - 5 } & Grosor $(\mathrm{cm})$ & $\begin{array}{c}\text { Pequeño } \\
\text { (Zacatecas) }\end{array}$ & $\begin{array}{c}\text { Intermedio } \\
\text { (Jalisco) }\end{array}$ & $\begin{array}{c}\text { Grande (San Luis } \\
\text { Potosí) }\end{array}$ \\
\hline \multirow{6}{*}{ Raíz } & $5-10.5$ & $10.5-13.5$ & $13.5-25$ \\
& Longitud (cm) & $13-35$ & $35-50$ & $50-104$ \\
& Peso $(\mathrm{g})$ & $84-950$ & $950-2050$ & $2050-3530$ \\
& Perímetro (cm) & $15-30$ & $30-42$ & $30-42$ \\
& Volumen $\left(\mathrm{cm}^{3}\right)$ & $570-1250$ & $1250-2250$ & $250-6671$ \\
& Número planta & $1-4$ & $4-8$ & $8-19$ \\
& Longitud (cm) & $200-325$ & $325-475$ & $475-675$ \\
& & & & algunas hasta 26 m \\
\hline
\end{tabular}

Agrupamiento de Cucurbita foetidissima Kunt, acorde a componentes morfológicos.

Es conveniente mencionar que algunas plantas sobresalieron en longitud de guía (más 26 m) a pesar de las limitadas condiciones agroclimatológicas. Esta clasificación puede ser utilizada para proponer una forma de identificar, comparar poblaciones, seleccionar plantas en estado óptimo de crecimiento y complementar con las variables morfológicas según sea la finalidad de aprovechamiento agroindustrial de la planta (forraje, aceite, bioenergía, reforestación).

El análisis de los componentes morfológicos generó ocho grupos de plantas, determinando como componente principal, la amplitud de la raíz con $61 \%$ de la variabilidad y el largo del segundo entrenudo a lo largo de la guía con $22 \%$ de varianza adicional), la cual se dividió en dos vertientes correspondientes al contenido de número de guías y longitud de entrenudos (Cuadro 4 y 5).

La distribución de grupos en las diferentes regiones los grupos 1 y 6 prevalecen en casi todas las poblaciones de calabacilla loca. Lo anterior, es importante para ubicar de una manera rápida y sencilla poblaciones de calabacilla loca en un determinado lugar (Cuadro 5 y 6).

Cuadro 5. Grupos generados por caracteres morfológicos de la calabacilla loca.

\begin{tabular}{lccl}
\hline & Grupo & \multicolumn{1}{c}{ Descripción } \\
\hline Amplitud de raíz & Número de guías & VI & $\begin{array}{l}\text { Número intermedio de guías con raíz amplia y } \\
\text { tamaño normal }\end{array}$ \\
& II & $\begin{array}{l}\text { Número intermedio de guías y raíz amplia con } \\
\text { guías muy delgadas }\end{array}$ \\
& V & Gran cantidad de guías con raíz amplia \\
& IV & Cortos, con gran volumen de raíz \\
& VIII & Largos, con gran volumen de raíz \\
& VII & Tamaño intermedio y volumen pequeño de raíz \\
& III & Muy amplios con raíz amplia \\
& I & Amplios con raíz amplia \\
\hline
\end{tabular}


Cuadro 6. Las comunidades integradas a los grupos del 1 al 8.

\begin{tabular}{|c|c|c|c|c|c|c|c|c|}
\hline \multirow{2}{*}{ Comunidad } & \multicolumn{8}{|c|}{ Grupo } \\
\hline & I & II & III & IV & $\mathrm{V}$ & VI & VII & VIII \\
\hline \multicolumn{9}{|l|}{ Jalisco } \\
\hline Chinampas, Ojuelos & 2 & & & & & & & 1 \\
\hline Encarnación de Díaz & 1 & & 1 & 1 & & & & \\
\hline Lagos de Moreno & & & & & & & 3 & \\
\hline \multicolumn{9}{|l|}{ San Luis Potosí } \\
\hline El Centenario & & 1 & 1 & 1 & & & & \\
\hline La Cócona & 1 & & & & 1 & & & 1 \\
\hline Yoliatl & & & & 1 & 1 & 1 & & \\
\hline \multicolumn{9}{|l|}{ Zacatecas } \\
\hline Guadalupe & 1 & 1 & & & & & & 1 \\
\hline La Pendencia & 2 & & & & & & & 1 \\
\hline Ojocaliente & 1 & 1 & 1 & & & & & \\
\hline Total & 8 & 3 & 3 & 3 & 2 & 1 & 3 & 4 \\
\hline
\end{tabular}

\section{Conclusiones}

Existen diferencias morfológicas y etapas de crecimiento de plantas de calabacilla loca dependiendo de las regiones edafo-climáticas, siendo la mayor incidencia de luz solar, menor precipitación y mediana altitud sobre el nivel del mar como las condiciones óptimas para el mejor comportamiento. Las poblaciones de Yoliatl, Salinas de Hidalgo, San Luis Potosí, sobresalieron con mayor contenido de guías y frutos por planta, por lo cual, estas plantas pueden ser utilizadas como bancos de germoplasma para sus distintas aplicaciones (conservación de la diversidad o aprovechamiento agroindustrial sustentable).

Este es el primer reporte sobre poblaciones silvestre de calabacilla loca que indican su georeferenciación y estado de crecimiento y desarrollo para implementar una estrategia de aprovechamiento posible por los pobladores de la región.

\section{Agradecimientos}

Al Campus-SLP del Colegio de Postgraduados, por las facilidades para realizar los recorridos de campo a realizar los muestreos y a la Línea-3, Energía alterna y biomateriales del Colegio de Postgraduados, por el financiamiento de reactivos y materiales para realizar esta investigación.

\section{Literatura citada}

Anónimo. 1980. Carta de climas, Guadalajara 14 Q IV. Dirección General de Geografía del Territorio Nacional-Secretaría de Programación y Presupuesto (SPP). México, DF.

Anónimo. 2016. Documentos por municipio de los gobiernos de los estados de San Luis Potosí, Jalisco y Zacatecas. 
Calvo-Grajales, A. 2003. Extracción y purificación de aceite a partir de semilla de calabacilla. División de Ciencia Animal-Universidad Autónoma Agraria Antonio Narro (UAAAN). Saltillo, Coahuila. 130 p.

Cuanalo, C. H.; Ojeda, T. E.; Santos, O. A. y Ortiz, S. C. A. 1989. Provincias, regiones y subregiones terrestres de México. Centro de Edafología-Colegio de Postgraduados en Ciencias Agrícolas, Chapingo, Estado de México. 624 p.

Di Rienzo J. A.; Bajzarini, M.; González, L.; Casanoves, F. and Tablada, M. 2014. Unidad de Bioestadistica Infostat software estadístico. Registro dirección nacional de derechos de autor núm. 960318. CATIE, Costa Rica.

Ferrusquia-Villafranca, I. 1993. Geology of Mexico: a sinopsis. In: biologicaldiversity of México, origins anddistributions. Ramamoorthy, T. P.; Bye, R.; Lot, A.; Fa, J. (eds.). Oxford University Press, Oxford. 3-107 pp.

Flores, V. M. A. 2016. Plantas de zonas semiáridas del altiplano Centro-Norte de México con potencial para la producción de aceite. Tesis de Maestría en Ciencias. Colegio de Postgraduados en Ciencias Agrícolas. 86-87 pp.

García, E. 1973. Modificaciones al sistema de clasificación climática.

Granados-Sánchez, D. y López-Ríos, G. F. 1999. Un recurso forestal de zonas áridas: Calabacilla loca (Cucurbita foetidissima H.B.K.) Revista Chapingo Ser. Cienc. Forest. Amb. 5(1):35-40.

Hernández-González, M.; Calvo-Grajales, A.; Santana-Martínez, M. Á; Ruelas-Chacón, X. y Fuentes-Lara, L. O. 2000. Extracción y purificación de aceite a partir de semilla de calabacilla loca (Cucurbita foetidissima) para su aplicación en la industria alimentaria.

Hoover R. 2001. Composition, molecular structure, and physio chemical properties of tuber and root starches: a review. Carbo hydrate polymers. 45(3) 253-267.

Humán, Z. 1991. Descriptores de la batata. CIP/AVRDC/IBPGR.

Juárez-Pérez M. A; Reyes Agüero, J. A y Andrade-Aguilar, J. A. 1991. Flora útil de tres tipos de matorral en el altiplano Potosino-Zacatecano, México. Revista de Geografía Agraria.

Lira-Sade, R.; Eguiarte-Fruns, L.; Montés-Hernández, S. 2009. Proyecto recopilación y análisis de la información existente de las especies de los géneros cucúrbita y sechium que crecen y se cultivan en México.

Ordoñez-Morales L. P. 2007. Efecto de la época del año sobre la digestibilidad in situ del material seco de Cucurbita foetidissima, División de Ciencia Animal, UAAAN. Saltillo Coahuila, Méx. 32 p.

Tamayo, J. L. 1981. Geografía moderna de México. 9a edición. Trillas. DF, México. 382 p.

Villaseñor, R. J. L y Espinoza, F. J. 1998. Invasive plants: changing the land scape of America: factbook. Federal inter agency committee for the management of noxious and exotic weeds (FICMNEW). Washington, DC. USA. 109 p.

WebAcademia. 2013. http://centrodeartigos.com/articulos-enciclopedicos/article_84490.html. Tema: Cucurbita foetidissima, morfología y cultivo, distribución, utiliza y plagas. http://www.conabio.gob.mx/malezasdemexico/cucurbitaceae/cucurbita-foetidissima/ fichas/ficha.htm. 